\title{
1 Evolution of nestling faeces removal in avian phylogeny
}

2

3 Juan Diego Ibáñez-Álamo ${ }^{1,2^{*}}$, Enrique Rubio ${ }^{1}$ and Juan José Soler ${ }^{3}$

4

$5 \quad{ }^{1}$ Behavioral and Physiological Ecology Group, Centre for Ecological and Evolutionary

6 Studies, University of Groningen, Groningen, The Netherlands

$7 \quad{ }^{2}$ Department of Wetland Ecology, Estación Biológica de Doñana, C.S.I.C., Sevilla,

8 Spain

$9 \quad{ }^{3}$ Estación Experimental de Zonas Áridas (CSIC), Almería, Spain

10 Received 23 August 2016

11 Initial acceptance 19 September 2016

12 Final acceptance 11 November 2016

13 MS number 16-00746R

14 Correspondence: J.D. Ibáñez-Álamo, RUG, FWN, Goederenontvangst, Afd. Animal

15 Ecology, t.a.v. J.D. Ibáñez-Álamo, Nijenborgh 4, 9747AG, Groningen, The

16 Netherlands.

17 E-mail address: j.d.ibanez-alamo@,rug.nl

19 Nest sanitation-related traits have often been explained at the intraspecific level as reducing the probability of infection or detection by predators and parasites, but its

21 evolution within the avian phylogeny is still poorly understood. We compiled detailed 22 information of such traits for more than 400 bird species and, by means of modern comparative methodologies, we reconstructed the evolution of adults' contribution to removing their offspring's faeces and the production of faecal sacs by nestlings.

25 Furthermore, because the functional hypotheses used to explain nest sanitation 
26 behaviour assume potential effects of brood size, body mass, nestling period and diet,

27 we explored the association between these traits and those related to nest sanitation in a 28 phylogenetically controlled framework. Our results suggest that parental removal of 29 nestling faeces has driven the evolution of faecal sacs, while the ancestral states 30 involved birds with faecal sacs removed by parents. These results support the long-held 31 idea that faecal sacs facilitate the removal of faeces by parents. Moreover, we found that 32 animal diets and small body sizes have favoured the evolution of faecal sacs suggesting 33 the existence of some chemical and physical constraints in relation to the evolution of 34 the mucous covering. Our results highlight the importance of nest sanitation in the 35 evolution of birds and their life history characteristics.

37 Keywords: birds, faeces, faecal sacs, nest sanitation, parent-offspring relationships 
Nest sanitation behaviour is an important and widespread behaviour in birds that, despite being known for a long time (Blair \& Tucker, 1941; Herrick, 1900; Skutch, 1976; Thomson, 1934), is still poorly understood, particularly regarding its evolution (Gow, Wiebe, \& Musgrove, 2015; Guigueno \& Sealy, 2012; Lang, Straight, \& Gowaty, 2002). This is surprising because strategies and traits related to nest sanitation, or even the effort devoted to such activities, have been suggested to help infer levels of selection pressures acting within species-specific nest environments (Ibáñez-Álamo, RuizRodríguez, \& Soler, 2014). Recent experimental studies have focused on investigating adaptive values of removal by parents of nestling faeces, the most common form of nest sanitation behaviour in birds (Guigueno \& Sealy, 2012). Since faeces contain potentially pathogenic microorganisms, its removal would reduce the probability of infection (Ibáñez-Álamo, Ruiz-Rodríguez, et al., 2014). In addition, parasites and nest predators might use chemical (i.e. odours) and/or visual cues of nestling faeces to located active nests and, therefore, removing it from nests would reduce the probability of nest predation (Ibáñez-Álamo, Ruiz-Raya, Roncalli, \& Soler, 2014; Ibáñez-Álamo, Sanllorente, Arco, \& Soler, 2013; Petit, Petit, \& Petit, 1989; Weatherhead, 1984) and parasitism (Ibáñez-Álamo, Ruiz-Raya, Rodríguez, \& Soler, 2016). Nestlings of many species encapsulate faeces within a mucous covering forming faecal sacs (Blair \& Tucker, 1941; Herrick, 1900; Thomson, 1934; Weatherhead, 1984). This covering acts as a physical barrier to impede microbial infections of birds (Ibáñez-Álamo, RuizRodríguez, et al., 2014) and might facilitate manipulation and removal of faeces by parents (White 1773, cited in Blair \& Tucker, 1941; Blair \& Tucker, 1941; Herrick, 1900; Pycraft, 1909; Thomson, 1934). Thus, the evolution of faecal sacs should be associated with parental removal of faeces from nests, although this prediction has never been tested. Either parental removal would more easily evolve in species with 
nestlings producing faecal sacs, or the evolution of faecal sacs would be particularly beneficial in species in which parents removed them.

Benefits associated with nest sanitation behaviour may depend on ecological and life history characteristics. It is, for instance, possible that the costs associated with the presence of nestling faeces in the nest depend on the volume and contents of faeces. Everything else being equal, negative impacts of small faeces in terms of attracting nest predators or vectors for potentially dangerous microorganisms/parasites would be lower than those of larger faeces (Ibáñez-Álamo et al., 2016; Petit et al., 1989). Moreover, if faeces are not removed and accumulate in the nest during nestling development, species with longer nestling periods and larger broods would differentially suffer higher costs than species with the opposite life history traits. Therefore, we expect species with longer nestling periods or larger broods to be those with faeces removal.

Species-specific diet is another element that might have influenced the evolution of nest sanitation-related traits (Guigueno \& Sealy, 2012). Diet affects the prevalence of potentially pathogenic microorganisms in chicken faeces (Ryu, Park, Bang, Kang, \& Hwangbo, 2016) and the presence of animal components in the food of livestock produces faeces with more intense odours than those feeding only from plants (e.g. Mackie, Stroot, \& Varel, 1998). Consequently, there are good reasons to think that diet characteristics could affect the costs of not removing faeces from nests in terms of microbial infection or detectability by predators. We explored this possibility by analysing the relationship between diet (i.e. animals or plants) and nest sanitationrelated traits (faeces removal and faecal sac production).

Previous studies on the subject have been focused on exploring within-species variation in a small number of bird species (e.g. Herrick, 1900; Ibáñez-Álamo et al., 2016, 2013; Ibáñez-Álamo, Ruiz-Raya, et al., 2014; Ibáñez-Álamo, Ruiz-Rodríguez, et 
al., 2014; Petit et al., 1989; Quan, Li, Wang, \& Goodale, 2015; Thomson, 1934; Weatherhead, 1984), but exploring the interspecific associations among traits related to nest sanitation and ecological and life history characteristics is essential to understand the evolution of nest sanitation in birds (Gow et al., 2015). Trying to fill this gap, we investigated these scenarios potentially affecting the evolution of nest sanitation using information collected from the literature for more than 400 bird species (19 Orders).

\section{Methods}

\section{Data collection}

After checking reviews on the topic (Blair \& Tucker, 1941; Guigueno \& Sealy, 2012; Thomson, 1934), we searched for related articles in the Web of Science and Google Scholar by using the following keywords: 'sanitation', 'nest sanitation', 'nest cleaning' and 'f(a)ecal sacs'. We also checked the Handbook of the Birds of the World (Del Hoyo, Elliott, Sargatal, Christie, \& de Juana, 2016) for information on nest sanitation-related traits. The literature used for each species is listed in the Supplementary Material (Table S1). For each species, we gathered information on (1) whether faeces are removed from nests; we considered a species to show removal when adults and/or offspring in some way avoided its accumulation in the nest. We noted (2) the presence of faecal sacs and whether (3) parents and (4) offspring remove nestling faeces at least during part of the nestling period. If parents removed nestling faeces, we also noted (5) which sex (male, female or both) was responsible. For seven species (Accipiter nisus, Ardea cinerea, Buteo buteo, Chiroxiphia caudata, Falco peregrinus, Haliaeetus albicilla, Ocyceros birostris), we completed information for these variables using video recordings available on the Internet (ARKive.org). We did not include precocial species in our data 
112 set given that we were interested in the evolution of removing nestling faeces from nests.

We also collected data on (6) body mass, (7) brood size, (8) nestling period duration, (9) diet (animals versus plants) and (10) nesting habits (hole, semihole and open nesters) using the Handbook of the Birds of the World (Del Hoyo et al., 2016). We considered the diet of a species to be of animal origin when the nestlings were fed mainly with animals such as invertebrates (i.e. insects), vertebrates (i.e. birds) or carrion. Those species whose nestlings were mainly fed with seeds, fruits or nectar were classified as having plant diets. For those species without specific information on the nestling diet, we used the main feeding source for the species. All collected information is reported in Table S2.

\section{Statistical analyses}

Because the expected interspecific associations may have a strong phylogenetic component (Harvey \& Pagel, 1991) we used the phylogenetic association among the bird species considered in our analyses. Phylogenetic relationships were estimated in the Mesquite environment (Maddison \& Maddison, 2015) as the consensus (i.e. majority rules consensus) tree of 1000 phylogenetic trees downloaded from http://birdtree.org/ (Jetz, Thomas, Joy, Hartmann, \& Mooers, 2012; Table S3). The predicted associations were subsequently explored with phylogenetically controlled analyses.

Most of the characters we considered are of binary nature, including the contribution of adults and nestlings to removing faeces from nests. In most species (349 of 370) the removal of nestling faeces is only performed by parents, but in some, nestlings also contribute (11 species) or even are the only ones in charge of this task by 
137 directly disposing of their own faeces (10 species). Because of the limited number of species that do not remove faeces from the nests, or with nestlings participating in this task, we did not include more than one categorical independent factor in our model. As we were mainly interested in exploring the evolution of nest sanitation behaviour of adults in relation to nestling traits, we considered the binomial information of whether adults remove nestling faeces without the help of their offspring. Information on nestling diet was also compiled as binomial information (mainly animals versus mainly plants). In addition to binomial variables, some statistical models also include continuous independent factors, namely, body mass, nestling period and brood size. Thus, given that all dependent factors were of binomial distribution and the need to control phylogenetic relationships, we used a phylogenetic generalized linear mixed model for binary data (binaryPGLMM; Ives \& Garland, 2014; Ives \& Helmus, 2011) as implemented in the $\mathrm{R}$ (version 3.2.3. R Core Team, 2016) statistical environment with the appropriate libraries ('ape'; Paradis, Claude, \& Strimmer, 2004), 'MASS' (Venables \& Ripley, 2002) and 'mvtnorm' (Genz \& Bretz, 2009). The binaryPGLMM package performs linear regressions for binary phylogenetic data, estimating regression coefficients (hereafter 'estimate') with approximate standard error. At the same time, it estimates the strength of the phylogenetic signal in the residuals (hereafter 's') and gives an approximate conditional likelihood ratio test for the hypothesis that there is no signal (Ives \& Garland, 2014; Ives \& Helmus, 2011). direction of changes along the phylogenetic tree of several pairs of traits: (1) between parental contribution to removing faeces from nests (alone or not) and nestling production of faecal sacs, (2) between nestling diet (animals or plants) and adult contribution to removing faeces, and (3) between nestling diet and production of faecal 
sacs. Briefly, we used Pagel's discrete method to test models of independent and dependent evolution (Harvey \& Pagel, 1991). This method compares the ratio of likelihood of two models: one of the models where the rates of change in each character are independent of the state, and a second model where rates of change depend on the state of the other trait. Since likelihoods associated with each of the eight possibilities of transition are estimated, this approach provides a good method to study evolutionary pathways through estimations of transition rates between pairs of binary character states (i.e. test for any effects in Mesquite 3.04). In addition, we also tested for the possibility that character $\mathrm{X}$ depended on character $\mathrm{Y}$, or that character $\mathrm{Y}$ depended on $\mathrm{X}$. We performed these analyses as implemented in Mesquite (Maddison \& Maddison, 2015) with 50 ML replicates over 1000 repeated simulations.

\section{Results}

\section{Faeces removal by adults and presence of faecal sacs}

176 We collected information on nest sanitation-related characters of 417 species of birds 177 from 19 different Orders. Within the 396 species that remove nestling faeces from their nests ( $94.96 \%$ of all species analysed), reliable information on production of faecal sacs and on adult and nestling participation in faeces removal was available for 370 species. Most of these species (96.76\%) produce faecal sacs, and in most of them (97.49\%) only adults remove faeces from nests. In nests of the 12 species with no faecal sacs, faeces removal is mainly done by nestlings alone $(83.33 \%)$ although adults contribute in two of them. Another interesting result is that, when adults participate in faeces removal, both male and female perform this behaviour in the majority of species (91.9\%) and just in a few of them either the female $(6.8 \%)$ or the male $(1.3 \%)$ do it exclusively. 
tree suggests that the evolution of parental contribution to faeces removal from nests of their own faeces, evolved to nodes with faecal sacs and only adults removing faeces more than expected by random (Fig. 1). Therefore, the evolutionary acquisition of faecal sacs was more likely in nodes with adult removal and no nestling help (difference in log-likelihood $=2.92, P=0.029$ ), while the trait that only adults remove faeces depends on whether the ancestral species had faecal sacs (difference in log-likelihood $=$ 17.92, $P=0.047$; Fig. 1$)$.

\section{Nest sanitation, body mass, brood size and nestling period}

Nestling faeces removal was not significantly affected by body mass, brood size or nestling period (all binary PGLMM: $P>0.16$ ). In contrast, faecal sacs were more frequent in species of lower body mass (binary PGLMM: estimate (SE) = -3.48 (1.19), $Z=2.93, P=0.003$; phylogenetic signal s2: $6.52, P=0.001)$ and shorter nestling period (binary PGLMM: estimate $(\mathrm{SE})=-9.69(3.80), Z=2.55, P=0.011$ : phylogenetic signal s2: 7.12, $P=0.0003$ ). Brood size did not predict the existence of faecal sacs (binary PGLMM: $P=0.5221$ ) and, when considering all three life history traits together, body mass (binary PGLMM: $P=0.027$ ), but not the duration of the nestling period (binary PGLMM: $P=0.160$ ) or brood size (binary PGLMM: $P=0.408$ ), reached statistical significance explaining faecal sac evolution. When body mass and parental contribution to removing nestling faeces from nests (i.e. with no help from nestlings) were considered together, the presence of faecal sacs tended to be more common in species in which only adults remove faeces (binary PGLMM: $P=0.07$ ), while the effect of body 
mass reached statistical significance (binary PGLMM: $P=0.025$ ). Moreover, nestlings

help parents to remove faeces from nests more often in large species (binary PGLMM: estimate $(\mathrm{SE})=2.11(0.62), Z=3.40, P=0.0007$ : phylogenetic signal s2: $4.64, P=$ 0.0001). Species that do not remove nestling faeces are similarly distributed among hole $(6.3 \%)$, semihole $(1.9 \%)$ and open nesters $(4.9 \%)$ suggesting that is unlikely that nesting habits affected the evolution of this behaviour.

\section{Nest sanitation and nestling diet}

The behaviour of removing nestling faeces from the nest was not associated with offspring diet $(88.0 \%$ of the 50 species with plant diets removed faeces while $95.9 \%$ of the 363 species with animal diets did so; binary PGLMM: estimate $(\mathrm{SE})=0.01(0.83), Z$ $=0.01, P=0.99:$ phylogenetic signal s2: $3.56, P<0.0001)$. Nestlings of species feeding on plants more often produced faeces within a mucous covering than those eating mainly animals $(100 \%$ of the 32 species with plant diets produced faecal sacs while 96.2\% of the 313 species with animal diets did so; binary PGLMM: estimate (SE) = 7.56 (3.38), $Z=2.24, P=0.025)$, even after controlling for the effect of body mass (binary PGLMM: estimate $(\mathrm{SE})=-4.31(0.97), Z=4.45, P<0.0001$; phylogenetic signal of the model: $\mathrm{s} 2=2.29, P<0.0001)$. In contrast, species-specific nestling diets were not associated with parental removal of faeces (binary PGLMM: estimate $(\mathrm{SE})=$ 1.88 (1.72), $Z=1.09, P=0.27$ ), but were associated with body mass (binary PGLMM: estimate $(\mathrm{SE})=2.25(0.65), Z=3.44, P=0.0005$; phylogenetic signal of the model: $\mathrm{s} 2$ $232=5.013, P<0.0001)$. Results from correlations of the evolution of the characters considered showed that the production of faecal sacs (Fig. 2), but not parental contribution to remove faeces from nests (differences in log-likelihood $=2.62, P=$ 0.23 ), tended to be associated with nestling diet. We found evidence supporting the 
influence of nestling faecal sacs on diets (differences in log-likelihood $=5.76, P<$ 0.0001 ) and of diets on faecal sacs (differences in log-likelihood $=4.31, P=0.04$ ). Thus, diet is likely to be responsible for the evolution of faecal sacs but probably not for the role that adults play in nest sanitation behaviour.

\section{Discussion}

Adults of most altricial bird species remove their nestling faeces from their nests, and these faeces are usually encapsulated in a mucous covering that reduces the probability of bacterial infection (Ibáñez-Álamo, Ruiz-Rodríguez, et al., 2014) and, apparently, facilitates faecal handling by parents (White 1773, cited in Blair \& Tucker, 1941; Blair \& Tucker, 1941; Herrick, 1900; Pycraft, 1909; Thomson, 1934). Furthermore, there seems to be no differences between males and females as both sexes perform this behaviour in most species in which adults participate in the removal of nestling faeces. Our results show that these traits (adult removal and faecal sacs) are almost completely fixed in altricial birds and much more widespread than previously thought (Blair \& Tucker, 1941; Skutch, 1976; Thomson, 1934), which suggests that parental participation in cleaning the nest is critical for offspring development. Nest sanitation is an essential component of parental behaviour, but its functionality has only been studied intraspecifically (Carere \& Alleva, 1998; Dell'Omo, Alleva, \& Carere, 1998; Gow et al., 2015; Lang et al., 2002; Thomson, 1934). To our knowledge, this is the first interspecific study on the topic and the only one looking at the evolution of nest sanitation within the avian phylogeny. Our main findings are (1) the existence of correlated evolution between adult removal of faeces and the production of faecal sacs by nestlings and (2) the detection of significant associations between life history characteristics (i.e. nestling period and body mass), the evolution of faecal sacs and 
parental contribution to removing faeces from nests. Finally, we also found (3) evidence suggesting a role of nestling diet on the evolution of faecal sacs. All these results considered together suggest that nest sanitation behaviour of adults, together with nestling diet, have influenced the evolution of the structure of nestling faeces, particularly in relation to the presence of the mucous covering (faecal sacs). Below we discuss possible evolutionary implications of our findings.

Altriciality is considered the derived character of precocial ancestors (Starck \& Ricklefs, 1998) and selection pressures favouring the evolution of nest sanitation would be higher in altricial species whose nestlings develop within the nest environment (Guigueno \& Sealy, 2012). Because we were interested on the evolution of removing nestling faeces from nests, we did not include precocial species in our data set (apart from one semiprecocial species, Antigone antigone) and thus cannot discuss the possibility of correlated evolution between nest sanitation and altriciality. However, since adults removing nestling faeces and nestlings producing faecal sacs were the ancestral states (Fig. 1), we speculate that these traits had already evolved in precocial bird species. Nest sanitation behaviours occur not only during the nestling phase, but also during the egg incubation stage (Guigueno \& Sealy, 2012), for instance renovating green materials (Petit, Hossaert-McKey, Perret, Blondel, \& Lambrechts, 2002) or removing broken eggs or eggshells from the nest (Soler et al., 2011; Tinbergen et al., 1962). Associated benefits of nest sanitation should be higher during the nestling period and thus these traits would rapidly become fixed in altricial bird populations. However, that the presence of faecal sacs is the ancestral state and that we only found them in altricial species in our literature search also suggest that the mucous covering might have evolved in relation to altriciality. 

and nestling production of faecal sacs. Faecal sacs mainly evolved in species in which adults remove faeces from their nests without help of their nestlings, while faeces removal exclusively by adults evolved mainly in species with faecal sacs (Fig. 1). These associations would be explained by faecal sacs facilitating faeces removal by parents (White 1773, cited in Blair \& Tucker, 1941), an idea already mentioned in the 18th century and now widely accepted by the scientific community (Blair \& Tucker, 1941; Herrick, 1900; Pycraft, 1909; Thomson, 1934) even though it has not previously been demonstrated. Our results offer the first demonstration of this hypothesis and we encourage future studies to investigate whether predictions of the evolutionary pattern found here fit the ecological context by, for example, exploring intraspecific covariation in faecal sac resistance and the efficacy of parents removing nestling faeces.

We found no evidence supporting the expected associations between nestling faeces removal and life history characteristics (see above), but body size and duration of the nestling period were negatively associated with the presence of faecal sacs. We predicted the opposite direction, however, because nest sanitation tasks should be more beneficial for larger species (i.e. higher production of faeces) and for those with longer nestling periods (higher accumulation of faeces). When considering both variables in the same model, only body mass reached statistical significance. Explanations of these results might be related to physical constraints of the mucous covering: above a certain weight it may not guarantee the integrity of faecal sacs during parental removal. New data are necessary to test this and other possible scenarios explaining the detected association between body mass and faecal sac production. Independently of the reason, our results show that nestlings participated in nest sanitation tasks more often in large species (see Results), which may be related to the difficulty adults have removing faeces 
with a weak, or no, mucous covering. Thus, because of the adaptive value of nest sanitation (Kölliker, Royle, \& Smiseth, 2012), nestlings of large species should directly dispose of their own faeces from nests more often than those of small species.

We also argued that nestling diet should have affected the evolution of nest sanitation behaviour and we found partial support for this hypothesis because faecal sacs are more likely to evolve in species with nestlings feeding mainly on vegetal material (Fig. 2). Diet, however, does not seem to have affected the evolution of parental sanitation behaviour. Moreover, faecal sacs appeared less often in species with nestlings mainly fed with animals. This is the most common source of food in birds (72.4\%; calculated from Burin, Kissling, Guimarães, Şekercioğlu, \& Quental, 2016), and, above, we proposed various scenarios in which the mucous covering could provide fitness advantages for species with animal diets. We found the opposite result, however, since faecal sacs appear more often in species with vegetal diets. We could speculate that animal diets favour faeces with some specific components or in such concentrations (i.e. uric acids) that might negatively interact with the mucous covering, but our results do not allow us to discuss this or other scenarios (i.e. detectability by predators and/or parasites). Thus, although our results emphasize the importance of nestling diet for the evolution of nest sanitation behaviour (i.e. faecal sacs), further investigation is necessary to detect the underlying causes.

Summarizing, our comparative analyses support the hypothetical correlated evolution of parent and offspring traits related to nest sanitation, and emphasize the importance of nest sanitation in the evolution of birds in general and their life history characteristics in particular. We hope that our findings encourage further research directed to explore functional hypotheses of the detected evolutionary patterns. 
336 We thank all the people that have collected the information on nest sanitation over the 337 years and that have made this study possible. We are also grateful to two anonymous referees whose interesting suggestions improved the manuscript. JDI has been financed by a postdoctoral contract (TAHUB-104) from the program "Andalucía Talent Hub" 340 (co-funded by the European's Union Seventh Framework Program Marie SkłodowskaCurie actions (COFUND) and the regional Government of Andalucía). Funding was partially provided by the Spanish Ministerio de Economía y Competitividad (European funds (FEDER)) (CGL2013-48193-C3-1-P).

Supplementary Material

Supplementary material associated with this article is available, in the online version, at doi

References

Blair, R. H., \& Tucker, B. W. (1941). Nest sanitation. British Birds, 34, 206-255.

Burin, G., Kissling, W. D., Guimarães, P. R., Şekercioğlu, Ç. H., \& Quental, T. B. (2016). Omnivory in birds is a macroevolutionary sink. Nature Communications, 7, 11250. https://doi.org/10.1038/ncomms11250

Carere, C., \& Alleva, E. (1998). Sex differences in parental care in the common swift (Apus apus): Effect of brood size adn nestling age. Canadian Journal of Zoology, 76(7), 1382-1387.

Del Hoyo, J., Elliott, A., Sargatal, J., Christie, D., \& de Juana, E. (2016). Handbook of the Birds of the World Alive. Barcelona, Spain: Lynx Edicions. 
Dell'Omo, G., Alleva, E., \& Carere, C. (1998). Parental recycling of nestling faeces in the common swift. Animal Behaviour, 56, 631-637. https://doi.org/10.1006/anbe.1998.0839

Genz, A., \& Bretz, F. (2009). Computation of multivariate normal and t probabilities. Psychological Science (Vol. 25). Heidelberg, Germany: Springer Verlag. https://doi.org/10.1007/s13398-014-0173-7.2

Gow, E. A., Wiebe, K. L., \& Musgrove, A. (2015). Nest sanitation in response to shortand long-term changes of brood size: Males clean more in a sex-role-reversed species. Animal Behaviour, 104, 137-143. https://doi.org/10.1016/j.anbehav.2015.03.014

Guigueno, M. F., \& Sealy, S. G. (2012). Nest sanitation in passerine birds: Implications for egg rejection in hosts of brood parasites. Journal of Ornithology, 153(1), 3552. https://doi.org/10.1007/s10336-011-0731-0

Harvey, P. H., \& Pagel, M. D. (1991). The comparative method in evolutionary biology. Oxford Series in Ecology and Evolution. Oxford, U.K.: Oxford University Press.

Herrick, F. H. (1900). Care of nest and young. Auk, 17, 100-103.

Ibáñez-Álamo, J. D., Ruiz-Raya, F., Rodríguez, L., \& Soler, M. (2016). Fecal sacs attract insects to the nest and provoke an activation of the immune system of nestlings. Frontiers in Zoology, 13(1), 3.

Ibáñez-Álamo, J. D., Ruiz-Raya, F., Roncalli, G., \& Soler, M. (2014). Is nest predation an important selective pressure determining fecal sac removal? The effect of olfactory cues. Journal of Ornithology, 155(2), 491-496. https://doi.org/10.1007/s10336-013-1031-7

Ibáñez-Álamo, J. D., Ruiz-Rodríguez, M., \& Soler, J. J. (2014). The mucous covering of fecal sacs prevents birds from infection with enteric bacteria. Journal of Avian 
Ibáñez-Álamo, J. D., Sanllorente, O., Arco, L., \& Soler, M. (2013). Does nest predation risk induce parent birds to eat nestlings' fecal sacs? An experimental study. Annales Zoologici Fennici, 50(1-2), 71-78. https://doi.org/10.5735/086.050.0106

Maddison, W. P., \& Maddison, D. R. (2015). Mesquite: a modular system for

Ives, A. R., \& Garland, T. (2014). Phylogenetic regression for binary dependent variables. In L. Garamszegi (Ed.), Modern phylogenetic comparative methods and their application in evolutionary biology (pp. 231-261). New York, NY: Springer. https://doi.org/10.1007/978-3-662-43550-2_9

Ives, A. R., \& Helmus, M. R. (2011). Generalized linear mixed models for phylogenetic analyses of community structure. Ecological Monographs, 81(3), 511-525. https://doi.org/10.1890/10-1264.1

Jetz, W., Thomas, G. H., Joy, J. B., Hartmann, K., \& Mooers, A. O. (2012). The global diversity of birds in space and time. Nature, 491(7424), 444-448. https://doi.org/10.1038/nature11631

Kölliker, M., Royle, N., \& Smiseth, P. (2012). Parent-offspring co-adaptation. In N. Royle, P. Smiseth, \& M. Kölliker (Eds.), The evolution of parental care. (pp. 285299). Oxford, U.K.: Oxford University Press.

Lang, J. D., Straight, C. A., \& Gowaty, P. A. (2002). Observations of fecal sac disposal by Eastern Bluebirds. Condor, 104(1), 205-207.

Mackie, R. I., Stroot, P. G., \& Varel, V. H. (1998). Biochemical identification and biological origin of key odor components in livestock waste. Journal of Animal Science, (76), 1331-1342. evolutionary analysis. Version 3.04. http://mesquiteproject.Org.

Paradis, E., Claude, J., \& Strimmer, K. (2004). APE: Analyses of phylogenetics and 
evolution in R language. Bioinformatics, 20(2), 289-290. https://doi.org/10.1093/bioinformatics/btg412

412

413

414

415

Petit, C., Hossaert-McKey, M., Perret, P., Blondel, J., \& Lambrechts, M. M. (2002). Blue tits use selected plants and olfaction to maintain an aromatic environment for nestlings. Ecology Letters, 5(4), 585-589. https://doi.org/10.1046/j.14610248.2002.00361.x

Petit, K. E., Petit, L., \& Petit, D. R. (1989). Fecal sac removal: Do the pattern and distance of dispersal affect the chance of nest predation? The Condor, 91(January), 479-482. https://doi.org/10.2307/1368331

Pycraft, W. P. (1909). A History of Birds. London, U.K.: Methuen.

Quan, R.-C., Li, H., Wang, B., \& Goodale, E. (2015). The relationship between defecation and feeding in nestling birds: observational and experimental evidence. Frontiers in Zoology, 12(1), 21. https://doi.org/10.1186/s12983-015-0116-y

R Core Team. (2016). R: A language and environment for statistical computing. Vienna, Austria: R Foundation for Statistical Computing.

Ryu, S. T., Park, B. S., Bang, H. T., Kang, H. K., \& Hwangbo, J. (2016). Effects of antiheat diet and inverse lighting on growth performance, immune organ, microorganism and short chain fatty acids of broiler chickens under heat stress. Journal of Environmental Biology, 37(2), 185-192.

Skutch, A. F. (1976). Parent birds and their youngs. The Corrie Herring Hooks Series (Vol. 2). Austin, TX: University of Texas Press.

Soler, J. J., Peralta-Sánchez, J. M., Martínez-Bueno, M., Martín-Vivaldi, M., MartínGálvez, D., Vela, A. I., ... Pérez-Contreras, T. (2011). Brood parasitism is associated with increased bacterial contamination of host eggs: Bacterial loads of host and parasitic eggs. Biological Journal of the Linnean Society, 103(4), 836- 
436 Starck, J., \& Ricklefs, R. (1998). Patterns of development: the altricial-precocial

437

438

439

440

441

442

443

444

445

446

447

448

449

450

451

452

453

454

455

456

457

458 spectrum. In J. Starck \& R. Ricklefs (Eds.), Avian growth and development. Evolution within the altricial precocial spectrum (pp. 3-30). Oxford, U.K.: Oxford University Press.

Thomson, D. F. (1934). Some adaptations for the disposal of faeces. Proceedings of the Royal Society B: Biological Sciences, 46, 701-707. https://doi.org/10.1017/CBO9781107415324.004

Tinbergen, N., Broekhuysen, G. J., Feekes, F., Houghton, J. C. W., Kruuk, H., \& Szulc, E. (1962). Egg shell removal by the Black-Headed Gull, Larus ridibundus L.; a behaviour component of camouflage. Behaviour, 19(1), 74-116. https://doi.org/10.1163/156853961X00213

Venables, W. N., \& Ripley, B. D. (2002). Modern Applied Statistics with S (Fourth Edi). New York, NY: Springer.

Weatherhead, P. J. (1984). Fecal sac removal by Tree Swallows: The cost of cleanliness. The Condor, 86(2), 187-191. https://doi.org/10.2307/1367039 .

2

3

44


462 Figure legends

463

471 Fig 1: Flow diagram showing correlated evolution between the production of faecal sacs

472 by nestlings (faecal sac versus no faecal sac) and faeces removal by adults (only adults

473 versus nestlings). The number associated with each arrow represents the maximum

474 likelihood value and, together with arrow thickness, indicates which transition is

475 expected to be the most common. Probability $(P)$ in the centre of the diagram indicates

476 the probability of correlated evolution between the binary traits after 10 ML replicates

477 and 1000 repeated simulations. Parsimony ancestral states of characters are shown in 478 the grey box.

Fecal sacs and feces removal

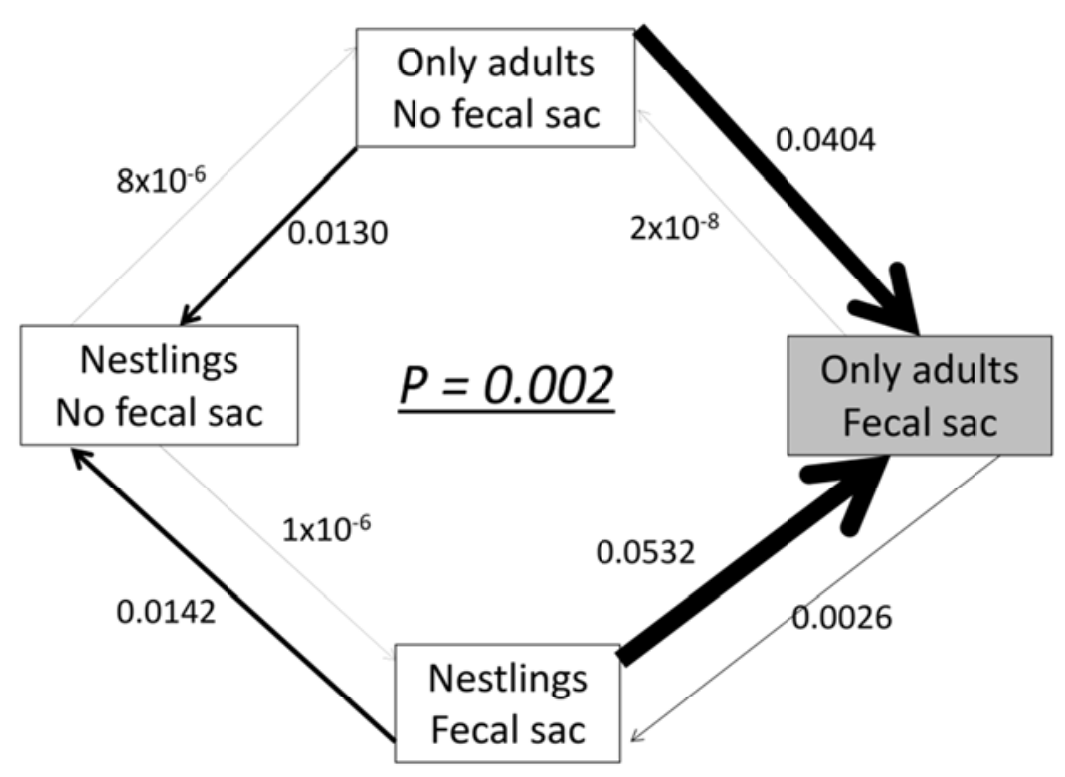


Fig 2: Flow diagram showing correlated evolution between the production of faecal sacs

481 by nestlings (faecal sac versus no faecal sac) and diet (animals versus plants). The

482 number associated with each arrow represents the maximum likelihood value and,

483 together with arrow thickness, indicates which transition is expected to be the most

484 common. Probability $(P)$ in the centre of the diagram indicates probability of correlated

485 evolution between the binary traits after $10 \mathrm{ML}$ replicates and 1000 repeated

486 simulations. Parsimony ancestral states of characters are shown in grey lboxes.

481

482

\section{Fecal sacs and diets}

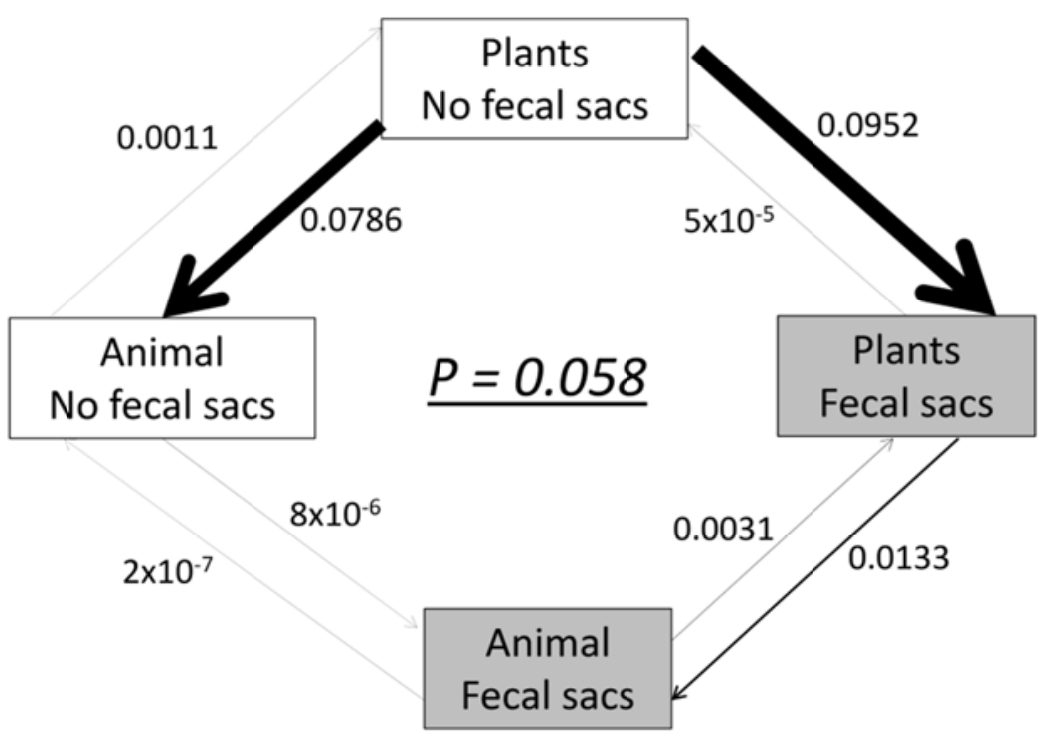

483 